\title{
Betamethasone versus dexamethasone on the fetal movement index in the women with preterm labor: A cross- sectional study
}

\author{
Abdolmajid Taheri $^{\circledR}$, Sheida Shabanian ${ }^{2 *}{ }^{(\mathbb{D}}$, Mohsen Khosravi $^{(\mathbb{D}}$, Soleiman Kheiri ${ }^{\circledR}$ \\ ${ }^{1}$ Assistant Professor, Department of Radiology, School of Medicine, Shahrekord University of Medical Sciences, Shahrekord, \\ Iran \\ ${ }^{2}$ Assistant Professor, Department of Gynecology and Obstetrics, Shahrekord University of Medical Sciences, Shahrekord, Iran \\ ${ }^{3} \mathrm{MD}$, Student Research Committee, Shahrekord University of Medical Sciences, Shahrekord, Iran \\ ${ }^{4}$ Professor of Biostatistics, Department of Epidemiology and Biostatistics, School of Health, Modeling in Health Research \\ Center, Shahrekord University of Medical Sciences, Shahrekord, Iran
}

*Corresponding Author: Sheida Shabanian, Department of Gynecology and Obstetrics, School of Medicine, Shahrekord University of Medical Sciences. Tel: +989131858493. Email: sheidashabanian@yahoo.com

\begin{abstract}
Background and aims: Biophysical evaluation of fetal profile and movements is a useful tool for assessing fetal health, which even has significant effects on neonatal health. This study aimed to compare the biophysical parameters of the profile - fetal movement index, in particular - before and after corticosteroid administration in two groups receiving dexamethasone and betamethasone.

Methods: In this descriptive/analytical study, 200 patients diagnosed with preterm delivery and admitted to the gynecology section of Hajar Hospital in Shahrekord in 2016 were investigated. Their demographic findings and medical histories were collected using a checklist form. Then their biophysical profiles 24 to 48 hours after corticosteroid administration were assessed. Finally, the data were analyzed by SPSS v. 18 software using $t$ test, chi-square and Fisher exact test.

Results: According to our study results, there was a significant difference in fetal movements before and after receiving corticosteroids, and the decrease in the percentage of movements was greater in the betamethasone group $(P<0.001)$. Also, the biophysical profile was significantly different in the dexamethasone group compared to the betamethasone group $(P<0.001)$. No significant difference was found between two groups in terms of fetal respiration and non-stress test (NST) before and after receiving corticosteroids $(P=0.47$ and $P=0.65$, respectively).

Conclusion: Dexamethasone administration had less effect on reducing fetal movement index than betamethasone administration. As the result, the biophysical profile was significantly different in the dexamethasone group compared to the betamethasone one.

Keywords: Fetal movement, Betamethasone, Biophysical profile, Dexamethasone, Preterm delivery
\end{abstract}

Received: 23 June 2021, Accepted: 2 August 2021, ePublished: 26 January 2022

\section{Introduction}

The rate of premature births before normal gestational age (i.e., from 20 to 37 weeks gestation) has recently increased among pregnant women (1). This increase has raised considerable concerns since it can cause numerous problems for both mothers and neonates or infants (2). Premature birth is one of the leading causes of death among non-abnormal infants, which places great economic and psychological burden on society $(3,4)$. Premature infants may experience fatal problems such as respiratory distress syndrome, intraventricular hemorrhage, patent ductus arteriosus, pneumothorax, and necrotizing inflammation of the gastrointestinal tract; however, the administration of glucocorticoids 48 hours or seven days before delivery can reduce the severity of these problems and disorders $(5,6)$. Previous studies have shown that prenatal glucocorticoid intake reduces fetal activity, behavioral changes, as well as psychological problems, and alters the biophysical profile score (7-9). Previous studies have also revealed that normal biophysical profile score in pregnancy leads to favorable pregnancy outcomes and, as a health indicator, reduces problems such as low APGAR scores, neonatal resuscitation, neonatal intensive care admission, as well as neonatal resuscitation and mortality $(10,11)$. Biophysical profile assessment is a useful tool for assessing fetal health; several studies have also recommended performing a comparison between betamethasone and dexamethasone in order for reducing maternal and neonatal complications (12). Moreover, drugs with positive effects and minimal side effects should be selected when prescribing corticosteroids for pregnant mothers - including pulmonary puberty of the fetus in preterm delivery - to serve different purposes (13). However, further studies are required to develop a more accurate assessment of the ambiguous aspects of corticosteroid administration in other populations, dosage, risky and multiple pregnancies, and drug preference (14). Therefore, the present study aimed to examine the preference of betamethasone or

(C) 2022 The Author(s); Published by Shahrekord University of Medical Sciences. This is an open-access article distributed under the terms of the Creative Commons Attribution License (http://creativecommons.org/licenses/by/4.0), which permits unrestricted use, distribution, and reproduction in any medium, provided the original work is properly cited. 
dexamethasone when dealing with fetal movements and biophysical profile. It was hoped that this study would effectively contribute to improving the preterm delivery protocol.

\section{Materials and Methods}

In this cross-sectional (descriptive/analytical) study, the medical files of mothers having suffered from preterm labor pain and received treatment for preterm labor from 22 April, 2014 to 22 June, 2015 in Hajar Hospital of Shahrekord were included. Out of all available cases, 200 ones with gestational age 28-34 weeks and meeting the inclusion criteria were explored in the study. Pregnant women with following status were also included in our study: those admitted to labor and gynecology with a diagnosis of preterm delivery (according to Creasy criteria) (15); those in 28 to 34 weeks of pregnancy; the ones received corticosteroids for pulmonary maturation; those with normal biophysical profile scores before receiving corticosteroids; and those completely satisfied with participating in the study. On the other hand, maternal medical complications such as diabetes and preeclampsia, abnormal fetal heart rate, fetal abnormalities, biophysical profile score less than 10, and impaired fetal movement prior to corticosteroid injection were considered as exclusion criteria. The census sampling method was adopted in the present study. The reduction rates of fetal movement for the group receiving betamethasone and for the one receiving dexamethasone were assumed equal to $40 \%$ and 60 samples, respectively (16); and considering $95 \%$ confidence interval and $80 \%$ power of sample size in each group based on the following formula, the sample size is equal to 99 and in the whole study, it is equal to 198. For more accuracy, 100 individuals were included in each group using the following formula:

$$
\frac{2\left(Z_{1-\frac{\alpha}{2}}+Z_{1-\beta}\right)^{2} P(1-P)}{d^{2}}
$$

Following the medical files, 100 files belonged to those who had received betamethasone and 100 ones belonged to those who had received dexamethasone were selected by a gynecologist. To match the groups, the number of biophysicals performed by each radiologist for both groups was considered equal; all biophysical profiles examined in this study had been performed 24 to 48 hours after receiving corticosteroids. After selecting the files based on the checklist form and receiving corticosteroids, the information required for this study including age, gestational age, fetal movement index, fetal respiration, amniotic fluid index (AFI), fetal coordination, and non-stress test (NST) were extracted. Each patient's information such as age, gestational age, and biophysical profiles was recorded in a checklist after receiving corticosteroids including fetal movement, fetal respiration, fetal coordination, AFI, and NST.

Then the collected data were analyzed by SPSS v. 22 using $t$ test, chi-square, and Fisher exact test. A value less than 0.05 was considered as the level of significance.

Results

In this study, a total of 200 pregnant women in 28 to 34 weeks of pregnancy and hospitalized for preterm delivery were examined. There was no significant difference between the two groups in terms of maternal age $(P=0.776)$ and gestational age $(P=0.191)$ (Table 1$)$.

After administrating the drugs, a significant difference was observed between the two groups receiving betamethasone and dexamethasone in terms of fetal movement $(P<0.001)$. Dexamethasone administration significantly reduced fetal movements and biophysical profile $(P<0.001)$. However, the comparison between the two groups receiving betamethasone and dexamethasone showed no significant difference in terms of fetal respiration $(P=0.47)$ and fetal heart rate variability $(P=0.65)$ (Table 2 ). It should be noted that all patients in both groups were normal in terms of fetal tone and AFI.

\section{Discussion}

This study aimed to compare the effects of dexamethasone and betamethasone on fetal motility in biophysical profile in preterm delivery. According to the study results, a significant reduction in fetal movements was observed in the betamethasone group. Furthermore, the biophysical profile in betamethasone group was significantly lower than that of dexamethasone group. As for other variables, no significant difference was detected between the two groups. These results may have been attributed to the inherent properties of corticosteroids reported by numerous studies conducted in this field. The changes resulting from the administration of corticosteroid drugs have some effects that manifest themselves as a decrease in the biophysical profile. Examples include hypoxia and profound acidosis which affect the cerebral cortex and reduce fetal movement and respiration (17). Our study results were in line with the findings from a study by Mushkat et al where betamethasone was determined to decrease the fetal movements while it was observed by the ultrasonic testing and perceived by the mother. There was also a decrease in fetal breathing movements of

Table 1. Comparison of Maternal and Gestational Ages in the Two Groups Receiving Dexamethasone and Betamethasone

\begin{tabular}{lccccc}
\hline Variables & Groups & Min & Max & Mean \pm SD & P value \\
\hline \multirow{4}{*}{$\begin{array}{l}\text { Maternal age } \\
\text { Betamethasone }\end{array}$} & 18 & 40 & $27.13 \pm 5.181$ & \\
& Dexamethasone & 17 & 42 & $26.92 \pm 5.260$ & 0.776 \\
& Total & 17 & 42 & $27.03 \pm 5.209$ & \\
& Betamethasone & 28 & 34 & $32.30 \pm 1.542$ & \\
Gestational & Dexamethasone & 28 & 34 & $32.01 \pm 3.293$ & 0.191 \\
age & & & & & \\
& Total & 3.28 & 34 & $32.01 \pm 2.581$ & \\
& & & & &
\end{tabular}


Table 2. Comparing the Effects of Dexamethasone and Betamethasone on Biophysical Parameters

\begin{tabular}{|c|c|c|c|c|c|c|}
\hline \multirow{2}{*}{ Biophysical parameters } & & \multicolumn{2}{|c|}{ Betamethasone } & \multicolumn{2}{|c|}{ Dexamethasone } & \multirow{2}{*}{$P$ value } \\
\hline & & Number & $\%$ & Number & $\%$ & \\
\hline \multirow{2}{*}{ Fetal movement } & Less than 3 moves $(0)$ & 35 & 35 & 9 & 9 & \multirow{2}{*}{$<0.001$} \\
\hline & Minimum 3 moves (2) & 65 & 65 & 91 & 91 & \\
\hline \multirow{2}{*}{ Fetal respiration } & 0 & 3 & 3 & 5 & 5 & \multirow{2}{*}{0.47} \\
\hline & 2 & 97 & 97 & 95 & 95 & \\
\hline \multirow{2}{*}{ Fetal heart rate variability } & 0 & 2 & 2 & 3 & 3 & \multirow{2}{*}{0.65} \\
\hline & 2 & 98 & 98 & 97 & 97 & \\
\hline \multirow{3}{*}{ Biophysical professional } & 6 & 4 & 4 & 7 & 7 & \multirow{3}{*}{$<0.001$} \\
\hline & 8 & 32 & 32 & 3 & 3 & \\
\hline & 10 & 64 & 64 & 90 & 90 & \\
\hline
\end{tabular}

those receiving betamethasone (18). In a similar study, dexamethasone was found to have fewer side effects in terms of fetal movement. Moreover, a significant reduction in the biophysical profile score was reported for betamethasone group, but not for the dexamethasone one (19). In Khazardoost et al study, betamethasone administration was discovered to cause a significant reduction in biophysical score, umbilical artery indices, and fetal movements (20). According to the results from a review study, there was a reduction in fetal motility, amniotic fluid volume, fetal respiration, and fetal heart rate after administrating betamethasone and dexamethasone (21). Taking into account the biophysical profile scores and movement counts, Jackson et al also reported that breathing motion and fetal movements were decreased after glucocorticosteroid administration. The decrease in AFI observed after administrating glucocorticosteroid may have been due to the reduction in the flow of alveolar fluid into the amniotic sac as well as the decrease in fetal respiration (8). In contrast, another study found that although administrating betamethasone to pregnant women resulted in evident yet transient reduction in fetal movement, the administration may have not necessarily and adversely affected the fetal health. Moreover, biophysical indicated that biophysical profile results of amniotic fluid were unchanged (22). The discrepancies observed in the results from the different studies could have been attributed to the differences in the type of studies, as well as to the transient changes in biophysical condition of the fetus. In general, it seemed that corticosteroids were capable of reducing the biophysical profile of the fetus by causing hypoxia and changes in the middle cerebral artery and by reducing intracranial pressure in the fetus $(23,24)$.

\section{Conclusion}

It was concluded that the administration of dexamethasone to pregnant women had a minimal reducing effect on fetal movement index and, therefore, on biophysical profile. Moreover, a significant decrease was observed in the betamethasone group regarding biophysical profile and fetus movement. Therefore, dexamethasone was found to be a more effective drug than betamethasone for pregnant women. Taking into account some factors such as the pharmacological side effects of each drug, the patients' conditions, as well as the findings obtained from the studies on pharmacological properties of corticosteroid drugs and their differences, however, it was highly recommended that further studies with larger sample sizes be conducted.

\section{Acknowledgments}

This article was derived from a research project approved by the Research and Technology Deputy of the Shahrekord University of Medical Sciences (approval no. 1823). Hereby, the researchers gratefully thank the patients who participated in this study.

\section{Authors' Contributions}

Conception and design, acquisition of data, analysis and interpretation of the data were performed by AT, MK, and SS. Drafting of the manuscript was done by AT. Critical revision of the manuscript for important intellectual content and statistical analysis were conducted by SK. The final draft was approved by MK, AT, and SS. All authors read and approved the manuscript.

\section{Conflict of Interests}

Authors declare that they have no conflict of interests.

Ethical Approval

This study protocol was approved by Ethic Committee of Shahrekord University of Medical Sciences (IR.SKUMS.REC.1394.15).

\section{References}

1. Iams JD, Cebrik D, Lynch C, Behrendt N, Das A. The rate of cervical change and the phenotype of spontaneous preterm birth. Am J Obstet Gynecol. 2011;205(2):130.e1-130.e6. doi: 10.1016/j.ajog.2011.05.021.

2. Suman V, Luther EE. Preterm labor. In: StatPearls. Treasure Island, FL: StatPearls Publishing; 2021.

3. Muhe LM, McClure EM, Nigussie AK, Mekasha A, Worku B, Worku A, et al. Major causes of death in preterm infants in selected hospitals in Ethiopia (SIP): a prospective, cross-sectional, observational study. Lancet Glob Health. 2019;7(8):e1130-e8. doi: 10.1016/s2214-109x(19)30220-7.

4. Ahmadzadeh N, Rezapour A, Ghanavatinejad Z, Nouhi M, Karimi S, Saravani A, et al. Estimation of economic burden of preterm and premature births in Iran. Med J Islam Repub Iran. 2017;31:78. doi: 10.14196/mjiri.31.78.

5. Khanafer-Larocque I, Soraisham A, Stritzke A, Al Awad E, Thomas S, Murthy P, et al. Intraventricular hemorrhage: risk factors and association with patent ductus arteriosus treatment in extremely preterm neonates. Front Pediatr. 2019;7:408. doi: 10.3389/fped.2019.00408.

6. Wynne K, Rowe C, Delbridge M, Watkins B, Brown K, 
Addley J, et al. Antenatal corticosteroid administration for foetal lung maturation. F1000Res. 2020;9. doi: 10.12688/ f1000research.20550.1.

7. Kelly MK, Schneider EP, Petrikovsky BM, Lesser ML. Effect of antenatal steroid administration on the fetal biophysical profile. J Clin Ultrasound. 2000;28(5):224-6. doi: 10.1002/ (sici)1097-0096(200006)28:5<224::aid-jcu3 > 3.0.co;2-g.

8. Jackson JR, Kleeman S, Doerzbacher M, Lambers DS. The effect of glucocorticosteroid administration on fetal movements and biophysical profile scores in normal pregnancies. J Matern Fetal Neonatal Med. 2003;13(1):50-3. doi: 10.1080/ jmf.13.1.50.53.

9. Davis EP, Sandman CA, Buss C, Wing DA, Head K. Fetal glucocorticoid exposure is associated with preadolescent brain development. Biol Psychiatry. 2013;74(9):647-55. doi: 10.1016/j.biopsych.2013.03.009.

10. Manandhar BL, Giri K, Rana A. Fetal biophysical profile score and perinatal outcome. J Nepal Health Res Counc. 2013;11(25):269-72.

11. Jha S, Dangal G. Role of modified biophysical profile in high risk pregnancy in predicting fetal outcome. J Nepal Health Res Counc. 2020;18(3):401-5. doi: 10.33314/jnhrc.v18i3.2513.

12. Peaceman AM, Bajaj K, Kumar P, Grobman WA. The interval between a single course of antenatal steroids and delivery and its association with neonatal outcomes. Am J Obstet Gynecol. 2005;193(3 Pt 2):1165-9. doi: 10.1016/j.ajog.2005.06.050.

13. Brownfoot FC, Crowther CA, Middleton P. Different corticosteroids and regimens for accelerating fetal lung maturation for women at risk of preterm birth. Cochrane Database Syst Rev. 2008(4):CD006764. doi: 10.1002/14651858.CD006764.pub2.

14. Roberts D, Brown J, Medley N, Dalziel SR. Antenatal corticosteroids for accelerating fetal lung maturation for women at risk of preterm birth. Cochrane Database Syst Rev. 2017;3(3):CD004454. doi: 10.1002/14651858.CD004454. pub3.

15. Resnik R, Creasy RK, lams JD, Lockwood CJ, Moore T, Greene MF. Creasy and Resnik's Maternal-Fetal Medicine: Principles and Practice E-Book. Elsevier Health Sciences; 2008.

16. Rotmensch S, Lev S, Kovo M, Efrat Z, Zahavi Z, Lev N, et al. Effect of betamethasone administration on fetal heart rate tracing: a blinded longitudinal study. Fetal Diagn Ther. 2005;20(5):371-6. doi: 10.1159/000086815.

17. Lear CA, Koome ME, Davidson JO, Drury PP, Quaedackers JS, Galinsky R, et al. The effects of dexamethasone on post-asphyxial cerebral oxygenation in the preterm fetal sheep. J Physiol. 2014;592(24):5493-505. doi: 10.1113/ jphysiol.2014.281253.

18. MushkatY, Ascher-Landsberg J, Keidar R, Carmon E, Pauzner D, David MP. The effect of betamethasone versus dexamethasone on fetal biophysical parameters. Eur J Obstet Gynecol Reprod Biol. 2001;97(1):50-2. doi: 10.1016/s0301-2115(00)00498-x.

19. Ghasemi Tehrani H, Khani B, Komrani Z. Comparison of the effect of betamethasone versus dexamethasone on the amniotic fluid index in the women at risk of preterm labor. J Res Med Sci. 2014;19(12):1124-8.

20. Khazardoost S, Pooransari P, Mirzamoradi M. The effect of betamethasone on fetal movement, biophysical profile and fetal circulation in preterm fetuses. Life Sci J. 2012;9(4):19902.

21. Musavi S, Nikniaz L, Hoseinifard H, Hamzehzadeh A, Vazifekhah S. Do corticosteroids affect prenatal biophysical parameters? a systematic review and meta-analysis. Int J Women's Health Reprod Sci. 2020;8(3):259-64. doi: 10.15296/ijwhr.2020.42.

22. Abbasalizadeh S, Neghadan Pharabar Z, Abbasalizadeh F, Ghojazadeh M, Goldust M. Efficacy of betamethasone on the fetal motion and biophysical profile and amniotic fluid index in preterm fetuses. Pak J Biol Sci. 2013;16(22):1569-73. doi: 10.3923/pjbs.2013.1569.1573.

23. Babović I, Radojičić Z, Plešinac S, Kastratović Kotlica B, Sparić R. Direct intramuscular fetal or maternal antenatal corticosteroid therapy: short-time effects on fetal behavior and oxygenation: a comparative study. J Matern Fetal Neonatal Med. 2016;29(19):3213-7. doi: 10.3109/14767058.2015.1121229.

24. Weiner Z, Hamisa R, Fisher A, Bombard A, Shalev E. The effect of steroids on the fetal brain and umbilical blood flow and biophysical profile. Am J Obstet Gynecol. 2003;189(6 Suppl):S218. doi: 10.1016/j.ajog.2003.10.588. 